\title{
DI-3-n-Butylphthalide Promotes Cardiac Function, Inhibit Oxidative Stress and Myocardial Apoptosis of Chronic Heart Failure Mice via Stimulating Nrf2/HO-1 pathway
}

\section{Zhongyu Wang}

China-Japan Union Hospital of Jilin University

Yan Zhang

Beihua University

Chun Yang

Beihua University

Hongliang Yang ( $\sim$ yanghongliang@jlu.edu.cn )

China-Japan Union Hospital of Jilin University https://orcid.org/0000-0002-7654-4667

\section{Research Article}

Keywords: DI-3-n-Butylphthalide, heart failure, Nrf2/HO-1 signaling pathway

Posted Date: September 15th, 2021

DOl: https://doi.org/10.21203/rs.3.rs-834507/v1

License: (c) (1) This work is licensed under a Creative Commons Attribution 4.0 International License.

Read Full License 


\section{Abstract}

\section{Purpose}

Heart failure (HF) continues to threat the human health and plagues the world, however, there are limited effective drugs for HF. We aimed to investigate the protective effect of DI-3-n-Butylphthalide (NBP) on myocardial injury in heart failure mice, and to study regulation mechanisms with $\mathrm{Nrf} 2 / \mathrm{HO}-1 / \mathrm{Ca}^{2+}$ SERCA2a axis.

\section{Methods}

Sixty C57BL/6J mice were grouped into five groups using a random number table: sham group (Sham), Heart Failure model group (HF), Heart Failure+ NBP group (HN), Heart Failure+NBP+Nrf2 inhibitor (HNM), Heart Failure+ NBP + calmodulin-dependent protein kinase II (CaMK囚) antagonist, KN93 (HNK). The HF mice was prepared using left anterior descending coronary artery ligation. As animal model preparation, the heart function was detected using echocardiography. H\&E and MASSON trichrome staining were performed to identify myocardial injury; The apoptosis of myocardial was examined by TUNEL staining assay. The levels of different oxidative stress-related proteins were measured through ELISA assay ; The reactive oxygen species and Nrf2 expression in heart tissue were observed with immunofluorescence assay. The levels of SERCA2a, calmodulin, endoplasmic reticulum stress regulatory protein and Nrf2/HO1 protein were measured using western blotting.

\section{Results}

The results demonstrated that NBP can significantly promote heart function, relieve the injury and inhibit cell apoptosis, meanwhile, reduce ERS injury in heart failure mice through increasing SERCA2a level and reducing $\mathrm{Ca}^{2+}$ influx. Finally, NBP was demonstrated to reduce CaMK囚phosphorylation levels and decrease cAMP-response element binding protein phosphorylation levels, which suggested that NBP could also activate Nrf2/HO-1 signaling pathway.

\section{Conclusions}

This study identified that NPBs treatment promotes the cardiomyocytes ERS and alleviates myocardial injury in heart failure mice which is related with stimulating $\mathrm{Nrf} 2 / \mathrm{HO}-1$ signaling pathway, regulating $\mathrm{Ca}^{2+}-$ SERCA2a and reducing $\mathrm{Ca}^{2+}$ influx.

\section{Background}

Heart failure (HF) is the one of most general diseases for hospital admissions worldwide ${ }^{1}$. HF is also a chronic developing disease in which the heart can't effectively pump enough blood to meet the body's metabolic need for blood and oxygen ${ }^{2}$. HF was usually accompanied with different complex clinically pathophysiological syndromes in circulation and/or pulmonary circulation congestion, seriously affecting 
the quality of patients' life $\mathrm{e}^{3}$. However, Therefore, it is important to find out the effective prevention and treatment measures for ameliorating symptoms, lowing all-cause mortality and promoting patients' health.

The endoplasmic reticulum is the machine used for proteins' processes, fold, and transportation in human body ${ }^{4}$. When ischemia ${ }^{5}$, hypoxia, drugs or toxins ${ }^{6}$ affect the endoplasmic reticulum homeostasis, it will induce the accumulation misfolded proteins. The ER is the major intracellular calcium store, involved in a number of fundamental calcium signaling pathways ${ }^{7}$. The state of protein accumulation in the cavity or imbalance of calcium ion $\left(\mathrm{Ca}^{2+}\right)$ homeostasis lead to endoplasmic reticulum stress (ERS) ${ }^{8}$. When the endoplasmic reticulum stress response is severe or lasts for a long time, it may induce the apoptosis and cause cell death ${ }^{9}$. Sarcoplasmic reticulum calcium ion transport ATPase-2a (SERCA2a) regulates the contraction and relaxation of cardiomyocytes by participating in the calcium ion transport of cardiomyocytes to maintain calcium ion homeostasis ${ }^{10}$. Under endoplasmic reticulum stress, decreased activity or decreased expression of SERCA2a causes an imbalance of calcium ion homeostasis in cardiomyocytes, which leads to cardiomyocyte contractile dysfunction and heart failure ${ }^{11}$.

The nuclear factor erythroid 2-related factor 2/ heme oxygenase-1 (Nrf2/HO-1) signalling pathway performed an important role in promoting heart functions and regulating myocardial remodel ${ }^{12,13}$.

The ubiquitination of Nrf2 was reduced and full function of Nrf2 was increased during ERS, which is dependent on modification of $\mathrm{C} 273, \mathrm{C} 288$ of Keap 1 protein ${ }^{14}$. The promoted Nrf2 could up-regulate the $\mathrm{HO}-1$ level and promote the antioxidant effect of cardiomyocytes via combining with angiotensin II receptor blocker (ARB), suggesting that Nrf2 is a key factor for regulating cardiomyocyte apoptosis ${ }^{15,16}$. Therefore, it has high value in clinical research in investigating the effective compound in inhibiting Cardiomyocytes' ERS response and keeping the balance of myocardial calcium to enhance patients' heart functions and decrease mortality.

DI-3-n-Butylphthalide(NBP) is isolated from seeds of Apium graveolens ${ }^{17}$. NBP has been passed by FDA of China in ischemic strokes therapy in $2002^{18}$. Meanwhile, many studies reported that NBP relieve the symptoms of ischemic stroke, and contributed to the long-term recovery ${ }^{19,20}$. However, the underlying mechanism by how NBP promotes heart failure is still not completely clear. Therefore, in this study, we studied the therapeutic effect of NBP treatment on heart function and the relationship with ERS in heart failure mice, and further discoveried the underlying the relationship with Nrf2/HO-1 pathway.

\section{Materials And Methods}

\section{Laboratory animals and grouping}

Sixty C57BL/6J mice, male, seven weeks old, were purchased from Beijing Huafukang Laboratory Animal Technology Co., Ltd. Mice were breed in $24-26^{\circ} \mathrm{C} \otimes 40-70 \%$ humidity in the laboratory animal center of Jilin 
University. Mice were randomly divided into five groups $(n=15)$ : sham operation group $(\mathrm{SH})$, HF model group (HF), HF + NBP (HN), HF + NBP+ CaMK囚 (KN93) (HNKC), HF + NBP + Nrf2 inhibitor

$(\mathrm{HNM})$. This whole animal experiment was approval by Laboratory Animal Welfare and Ethics Committee of Jilin University (IACUC No.2019156).

\section{Mice HF model preparation}

The preparation of mice HF model was followed as this protocol: Except for mice in the SH group, mice in other group were performed with the method of constriction of abdominal aorta to prepare HF mouse models ${ }^{21}$. Firstly, mice were anesthetized by $1 \%$ pentobarbital sodium $(45 \mathrm{mg} / \mathrm{Kg})$ with intraperitoneal injection. Place mice in supine position on a surgery platform with a heating pad to maintain the body temperature. The abdominal region of the mice with an animal hair clipper and hair remover lotion were shaved for avoiding surgical contaminations. Open the abdominal cavity at the center of the abdomen for $2 \sim 3 \mathrm{~cm}$, bluntly strip the muscles, expose the abdominal aorta, place a 7-gauge surgical needle parallel to the abdominal aorta, ligate the aorta and the needle with 0-gauge silk thread, Then remove the surgical needle to confirm the aorta is unobstructed and the abdomen is closed. In the sham operation group, only the abdominal aorta was isolated, not ligated. Mice were injected intraperitoneally with 50,000 $U$ penicillin daily for 3 days after operation for infection prevention.

The mice of sham group were given the same amount of penicillin daily antibiotics after operation for 3 days. After surgery, mice in HN group were given NBP $(60 \mathrm{mg} / \mathrm{kg})$ daily by intragastric administration by tail vein injection. Besides, mice in the HNK were co-treated by the same amount $(60 \mathrm{mg} / \mathrm{kg})$ of NBP and CaMKIl antagonist (KN93) with injection via tail vein post-surgery for 15 days. Moreover, mice of HNM group were treated with Nrf2 inhibitor (ML385, MCE, USA) before intragastric administration of NBP for 15 days.

\section{Mice heart function detection}

Mice heart function was detected by Cardiac ultrasound. M-mode echocardiography detection was performed for examination. And measurement of thickness of left ventricular anterior wall (LVAW) , left ventricular posterior wall (LVPW) and diameters of left ventricular end-diastolic (LVEDD) and left ventricular end-systolic (LVEDs), left ventricular ejection fraction (LVEF) and left ventricular short-axis shortening rate (LVFS) were made. These results were measured for three cardiac cycles, and the average value was calculated as the final test result.

\section{Haematoxylin-eosin (H\&E) staining}

The formalin-fixed cardiac tissues were fixed in formalin solution. Then the tissues were gradually dehydrated in gradient ethanol, transparent in xylene, and embedded in paraffin. Then tissue samples were cut into $4 \mu \mathrm{m}$ sections. Following that, the slices were immersed into haematoxylin for 10 min, rinsed with 1 *PBS, then differentiated in $1 \%$ hydrochloric acid alcohol. Eosin-stained solution 
was stained for $30 \mathrm{~s}$. After that, slices were dehydrated, transparented and sealed. Finally, the slices were observed under microscope.

\section{Masson staining}

The slices containing heart tissue were deparaffinised and rehydrated with ethanol, and then stained in Regaud haematoxylin solution. Then slices were stained in Masson ponceau-acid solution for $15 \mathrm{~min}$, then differentiated in $1 \%$ molybdenum phosphate acid. The sections were directly transferred to aniline blue solution and stained, afterwards, sections were differentiated in $0.2 \%$ glacial acetic acid. Following with that, slices were dehydrated in $100 \%$ ethanol, rinsed in xylene and mounted in neutral resins. The slices were examined using a light microscope (Olympus, Tokyo, Japan).

\section{TUNEL staining}

TUNEL assay kit (Roche Diagnostics, Mannheim, Germany) was used for cell apoptosis detection . The protocol followed with detail instructions in kit. The sample were observed and detected with fluorescence microscope, then the apoptosis rate was calculated.

\section{Immunofluorescence assay (IFA)}

Post-deparaffinised slices were submerged in hydrogen peroxide solution, rinsed in PBS and put into $0.1 \mathrm{M}$ sodium citrate solution. After incubating with goat serum solution for $30 \mathrm{~min}$, the slices was added with the probe of ROS and Nrf2 antibody, following with incubation overnight at $4^{\circ} \mathrm{C}$. After washing in PBC, fluorescent dye-labelled secondary antibody was added to the sections and incubated at $37^{\circ} \mathrm{C}$ for 30 min in darkness. DAPI dye was use to stain cell nuclear. After slices mounted in mounting solution, the targeting proteins were observed using the fluorescence microscope.

\section{ELISA}

ELISA Kit (ab108816, Abcam) was used to detect the level of BNP and the ELISA Kit (CEA485Ra, USCN, China) was also performed to examine the level of NT-proBNP in serum. In addition, the ELISA kits of superoxide dismutases (SOD) (ab65354, Abcam), malondialdehyde (MDA) (ab238537, Abcam) and catalase (CAT) (ab83464, Abcam) were performed according to the manufacture's protocols.

\section{Western blotting}

Heart tissue of mice were homogenized with RIPA buffer (CST, USA) at 15,000 rpm for 30 seconds and centrifugated for $15 \mathrm{~min}$ at $15,000 \mathrm{~g}$ at $4{ }^{\circ} \mathrm{C}$. The protein concentration in the supernatant were determined by BCA assay. $30 \mu \mathrm{g}$ protein were loaded and seperated in 10-12\% SDS-PAGE gels under electrophoresis and transferred to PVDF membranes. After blocked in $5 \%$ skim milk, membranes were seperately incubated overnight at $4^{\circ} \mathrm{C}$ with primary antibodies against $\mathrm{Bcl}-2$ (3498, CST, USA), Bax (5023, CST, USA), CaMKII (13-7300, Abcam, USA), phospho-CaMKII (12716, CST, USA), cAMP response element-binding (CREB) (4820, CST, USA), p-CREB (9198, CST, USA), SERCA2a (4388, CST, USA), 
dihydropyridine receptor (ab232983, Abcam, USA), Cav1.2 (ab84814, Abcam, USA), Caspase-12 (2202, CST, USA), Nrf2 (12721, CST, USA), HO-1 (43966, Abcam, USA), thioredoxin (Trx-1) (2298, CST, USA), GRP78 (ab21685, Abcam, USA) and GAPDH (51742, CST, USA) as the internal control. Then, PVDF membrane was added with HRP-conjugated goat anti-rabbit secondary antibody (1:1000,5597, CST, USA) for $1 \mathrm{~h}$, the intensity of protein bands was visualized with SuperSignal ${ }^{\mathrm{TM}}$ West Pico PLUS Chemiluminescent Substrate (CAT\#3480, Thermo Fisher Scientific, USA)

\section{Statistical methods}

Results were analyzed by Graphpad Prism 8.0 and represented as mean \pm SD. To determine the statistic significant differences, un-paired Student's t-test was used for two group comparison, One-way analysis of variance (ANOVA) was performed followed by multiply comparisons. P value less than 0.05 was considered to be statistically significant different.

\section{Results}

\section{The protective effects of NBP on HF mice's heart}

We investigated the NBP effect on HF mice, we successfully prepared the mice HF model by constriction of adomen aorta. The results showed that in HF group, the left ventricular end-systolic (LVDs) and diastolic (LVDd) were significantly increased, LVEF and LVFS were reduced (Fig. 1A, $P<0.05$ ), the level of heart failure biomarker BNP and NT-proBNP in plasma were both increased (Fig. 1B, $P<0.05)$, The arrangement of myocardial fiber cells is disordered, myocardial fibers are rupture in HF group (Fig.1C, $\mathrm{P}<0.05)$ as compared with mice in Sham group. These results demonstrated that HF mice model was established successfully. Under NBP administration, the phenotypes in HF mice model were all reversed (Fig.1A-1C, P<0.05). Our data showed that NBP could significantly relieved the myocardial injury in Heart Failure mice, meanwhile, enhanced the left ventricular systolic and diastolic function.

\section{Treatment of NBP promte the Pathological injury}

To investigate the relationship between NBP and Oxidative stress injury in myocardium, firstly, we found the accumulation of ROS in HF mice (Fig. 2A), interestingly, NBP administration significantly reduce the content of ROS, decrease MDA level in plasma and increase the releasing of SOD and CAT (Fig. 2B). Moreover, Myocardial apoptosis rate was slow significantly promoted in HF group (Fig. 2C), Bax was down-regulated during heart failure mice. Furthermore, the anti-apoptotic factor $\mathrm{Bcl}-2$ was decreased (Fig. 2D). These pathological damages were relieved after NBP treatment on HF mice, suggesting that NBP relieve oxidative stress injury and decrease the cardiomyocyte apoptosis.

\section{The relationship of NBP with ERS homeostasis}

The over-activation of ERS was reported to stimulate sarcoplasmic reticulum dysfunction of $\mathrm{Ca}^{2+}$ uptaking and releasing, and cause intracellular $\mathrm{Ca}^{2+}$ burden, inducing cardiomyocyte apoptosis $\mathbb{\text { leading }}$ 
to myocardial contractile and diastolic function. To maintain $\mathrm{Ca}^{2+}$ homeostasis is related with SERCA2a expression. To explore the regulatory mechanism of SERCA2a under NBP treatment, we demonstrated the expression of SERCA2a was reduced in HF mice (Fig.3A), inhibited the SERCA activity and lead to $\mathrm{Ca}^{2+}$ transport disorder, resulting to inhibiting the level of calmodulin, DHPR and Cav1.2 (Fig. 3A). The overloading of $\mathrm{Ca}^{2+}$ stimulate $\mathrm{Ca}^{2+}$ binding with CaMK冈to enhance the phosphorylation of CaMK冈and CREB (Fig.3B), meanwhile, the levels of GRP78 and Caspase-12, ERS related proteins, were up-regulated in HF mice (Fig. 3C), implying that ERS can cause degradation of SERCA2a, resulting in decreased cardiac diastolic function. When NBP was treated, SERCA2a was up-regulated, the calcium-regulated proteins were activated, resulting in the inhibition of CaMK囚 and CREB phosphorylation, meanwhile, the ERS injury was also alleviated assessed by GRP78 and Caspase-12, suggesting that NBP inhibits ERS via stimulating $\mathrm{Ca}^{2+}{ }^{+}$SERCA2a signaling pathway in HF mice.

\section{NBP treatment could motivate the Nrf2/HO-1 pathway}

Maintaining equilibrium between oxidative and antioxidative components in the ER is the key to regulate the oxidative stress response in cardiomyocytes. As one of the most important regulators

in maintaining the ER balance, Nrf2 could transfer into cell nucleus and bind with ARB as HF occurrence (Fig. 4A), as the result of that, the release of $\mathrm{HO}-1$ and Trx1 were induced in a meanwhile (Fig. 4B). After the administration of NBP, Nrf2 (Fig. 4A),. The high cytoplasmic expression level of Nrf2 promoted the further increased expressions of its downstream proteins, speculating that NBP improves the ERS through activating Nrf2/HO-1 pathway of Heart Failure mice.

\section{CaMKIl antagonist inhibits the benefits of NBP on myocardium in HF mice}

The detailed mechanism of NBP treatment on calcium channels was also investigated, the CaMKX antagonist, KN93, was administered in mice before NBP treatment. Interestingly, the protective effects of NBP on myocardium in HF mice were suppressed (Fig.5). The results demonstrated that the distinct myocardial injury (Fig. 5A) and injury fibrosis (Fig. 5B) in mice, the promoted apoptosis rate (Fig. 5C) and decreased expression of BNP and NT-proBNP (Fig. 5D) of HNK group.

\section{$\mathrm{Nrf2}$ inhibitor suppresses the activation of $\mathrm{Ca}^{2+}$-SERCA2a}

We also investigated the relationship of Nrf2/HO-1 pathway with $\mathrm{Ca}^{2+} / \mathrm{CaMK}$ /CREB pathway, ML385, an Nrf2 inhibitor, was used to treat HF mice before NBP administration. $\mathrm{Ca}^{2+}$ overload was inhibited in HF mice after ML385 treatment. Moreover, the expression of SERCA2a (Fig. 6A) and calcium regulatory proteins (Fig. 6B) were down-regulated in the HNM group, therefore, CaMKII and CREB phosphorylation were activated. Nrf2 inhibitor could up-regulate the ERS regulatory factors (Fig. 6C). Our data demonstrated that the protective role of NBP on HF mice depends on the activation of $\mathrm{Nrf} 2 / \mathrm{HO}-1 / \mathrm{Ca}^{2+}$ SERCA2a axis. 


\section{Discussion}

NBP is a compound extracted from celery seeds ${ }^{18}$. NBP can also be obtained by chemical synthesis. It is an emerging therapeutic drug. A large number of cells' and animals' studies have shown that NBP can protect the brain from acute ischemic damage, reduce the area of cerebral infarction, inhibit platelet aggregation, and reduce neuronal apoptosis and oxidative damage ${ }^{18}$. Chinese researchers conducted two double-blind, randomized, multi-center trials, demonstrating that NBP has shown good therapeutic effects on stroke. Due to its safety and effectiveness, NBP has been approved by the National Food and Drug Administration in $2002^{18}$. Recently NBP was reported to play a preventive role on acute myocardial infarction, however, it is still unclear whether NBP has a protective effect on chronic heart failure. Therefore, in this study, mice model of chronic heart failure was constructed by surgery and post-surgery exercise. After the model was successfully established, NBP was given intragastric to explore the effects of NBP on the heart function, oxidative stress, cardiomyocytes and apoptosis in chronic heart failure mice.

Chronic heart failure is characterized by heart muscle weakness and diastolic dysfunction, as well as reduced cardiac output. The heart was unable to meet the needs of the body's tissue cell metabolism, and blocked venous blood return ${ }^{22}$. Changes in hemodynamics and neurohumoral, resulting in various symptoms and signs ${ }^{23}$. Epidemiological results reported that Patient survival rates over a five-year period with heart failure is similar to cancers, and a total of $40 \%$ of cardiovascular deaths are caused by heart failure ${ }^{24}$. Oxidative stress imbalance and cardiomyocyte apoptosis are the main pathological manifestations of chronic heart failure. Mitochondrial dysfunction during heart failure, a large amount of reactive oxygen species release, induce the production of MDA, and the content of SOD that can antagonize free radicals is significantly reduced, leading to Myocardial cell energy metabolism disorder and contractile function decrease, which eventually lead to myocardial cell apoptosis. In this study, NBP can improve the effect of HF on the systolic and diastolic heart function, and reduce cardiac muscle injury, alleviate fibrosis, reduce myocardial cell apoptosis, and inhibit oxidative stress damage caused by endoplasmic reticulum stress. These results demonstrated that NBP could regulate calcium channels to perform cardioprotective function.

Heart failure is caused by a dysfunctional calcium regulation of the myocardial cell in the $\mathrm{ER}^{25}$. Cardiomyocyte excitation-contraction coupling (ECC) uses $\mathrm{Ca}^{2+}$ as the second messenger. The calcium cycle mainly includes two main processes: sarcoplasmic reticulum (SR) calcium release and calcium recapture ${ }^{25}$. After the ECC process is over, the removal of $\mathrm{Ca}^{2+}$ of cardiomyocytes cytoplasm is caused by calcium pump (SERCA2a) on the SR to re-uptake $\mathrm{Ca}^{2+}$ back to the SR. When HF occurs, the expression of SERCA2a decreases, indicating that $\mathrm{Ca}^{2+}$ influx causes intracellular calcium overload, stimulating $\mathrm{Ca}^{2+}$ binding to CaMK囚, leading to phosphorylation of the substrate of CaMK》, promoting its autophosphorylation, and activated CaMKX can phosphorylate CREB, bound with a specific sequence of cyclic adenosine phosphate response elements, recruits RNA polymerase II, forming transcription complex, thus controlling the gene transcription ${ }^{10,11}$. In this study, Butylphthalide treatment could 
significantly enhance SERCA2a expression in HF mice model, thereby activating the Calmodulin DHPR and Cav1.2, while reducing the CaMK $\triangle$ and CREB phosphorylation. Under treatment with CaMKX antagonist, the regulative effect of NBP on $\mathrm{Ca}^{2+}-$ SERCA2a is inhibited, and the myocardial protection of $\mathrm{HF}$ mice model is inhibited, meanwhile, suggesting that NBP treatment activate $\mathrm{Ca}^{2+}{ }^{2+}$ SERCA2a to promote myocardial calcium and protect the myocardial tissue in HF mice, which is associated with ERS.

When the Ca2+-SERCA2a homeostasis of cardiomyocytes is destroyed, the body's oxidation system and antioxidant system are out of balance, and the body's oxidation capacity exceeds its antioxidant capacity, causing abnormal expression of some proteins such as GRP78 and caspase-12, which affects its completion of normal proteins ${ }^{26}$. When the function is folded, endoplasmic reticulum stress (ER Stress, ERS) occurs. Nrf2, as a core transcription factor that resists oxidative damage, can induce cell damage and cell dysfunction, such as necrosis or pyrolysis ${ }^{27}$. Studies have shown that as Nrf2 was inhibited, it can aggravate myocardial oxidative stress and apoptosis, leading to cardiac dysfunction, however, upregulating the level of Nrf2 promoted the left ventricular function in mice with heart failure and reduce cardiac hypertrophy $28,29,30$. In our research, through stimulation of ERS, the Nrf2 performed the nuclear translocation and was increased in the nucleus. After entering the cell nucleus, Nrf2 usually combines with another transcription CREB and start the transcription process. This is consistent with the promotion of CaMK囚 and CREB phosphorylation in HF mice as we found. As mice was treated with the Nrf2 inhibitor, the regulation function in $\mathrm{Ca}^{2+}$-SERCA2a homeostasis by NBP was inhibited. Based on these results, we conclude that NBP could protect the myocardium and improve the ERS of cardiomyocytes through activating $\mathrm{Nrf} / \mathrm{HO}-1 / \mathrm{Ca}^{2+}-\mathrm{SERCA} 2 \mathrm{a}$ axis.

\section{Declarations}

Funding

This study was supported by the Enterprise Fund (2020YX0215)

Conflict of Interests/Competing interests

The authors declare that they have no competing interests.

Availability of data and materials

The datasets used and analyzed during the current study are available from the corresponding author upon reasonable request.

Authors' contributions

Hongliang Yang and Chun Yang designed the whole study. Zhongyu Wang and Chun Yang performed the animal experiment and other biochemical and molecular biology experiment. Zhongyu Wang and Yan 
Zhang analyzed the data. Zhongyu Wang and Yan Zhang wrote the manuscript. All authors read the manuscript.

\section{References}

1. Rossignol, P., Hernandez, A. F., Solomon, S. D. \& Zannad, F. Heart failure drug treatment. The Lancet 393, 1034-1044 (2019).

2. Damy, T. [Heart failure, progress and challenges]. Soins 62, 20-21 (2017).

3. Rangaswami, J. et al. Cardiorenal Syndrome: Classification, Pathophysiology, Diagnosis, and Treatment Strategies: A Scientific Statement From the American Heart Association. Circulation 139, (2019).

4. Mei, Y., Thompson, M. D., Cohen, R. A. \& Tong, X. Endoplasmic Reticulum Stress and Related Pathological Processes. Journal of Pharmacological \& Biomedical Analysis 1, 1000107 (2013).

5. Zhao, G. et al. Berberine protects rat heart from ischemia/reperfusion injury via activating JAK2/STAT3 signaling and attenuating endoplasmic reticulum stress. Acta Pharmacol Sin 37, 354367 (2016).

6. Chung, J.-G. Wogonin triggers apoptosis in human osteosarcoma U-2 OS cells through the endoplasmic reticulum stress, mitochondrial dysfunction and caspase-3-dependent signaling pathways. Int J Oncol (2011) doi:10.3892/ijo.2011.1027.

7. Appenzeller-Herzog, C. \& Simmen, T. ER-luminal thiol/selenol-mediated regulation of $\mathrm{Ca} 2+$ signalling. Biochemical Society Transactions 44, 452-459 (2016).

8. Hetz, C. The unfolded protein response: controlling cell fate decisions under ER stress and beyond. Nat Rev Mol Cell Biol 13, 89-102 (2012).

9. Jing, L. \& Jia, X. Lycium barbarum polysaccharide arbitrates palmitate-induced apoptosis in MC3T3-E1 cells through decreasing the activation of ERS-mediated apoptosis pathway. Mol Med Report (2017) doi:10.3892/mmr.2017.8128.

10. Asahi, M. et al. Cardiac-specific overexpression of sarcolipin inhibits sarco(endo)plasmic reticulum Ca2+ ATPase (SERCA2a) activity and impairs cardiac function in mice. Proceedings of the National Academy of Sciences 101, 9199-9204 (2004).

11. Ferrandi, M. et al. Istaroxime stimulates SERCA2a and accelerates calcium cycling in heart failure by relieving phospholamban inhibition: Istaroxime stimulation of SERCA2a in heart failure. $\mathrm{Br} \mathrm{J}$ Pharmacol 169, 1849-1861 (2013).

12. Li, H. et al. Paeonol and danshensu combination attenuates apoptosis in myocardial infarcted rats by inhibiting oxidative stress: Roles of Nrf2/HO-1 and PI3K/Akt pathway. Sci Rep 6, 23693 (2016).

13. Li, J., Ichikawa, T., Janicki, J. S. \& Cui, T. Targeting the Nrf2 pathway against cardiovascular disease. Expert Opinion on Therapeutic Targets 13, 785-794 (2009).

14. Wakabayashi, N. et al. Protection against electrophile and oxidant stress by induction of the phase 2 response: Fate of cysteines of the Keap1 sensor modified by inducers. PNAS 101, 2040-2045 
(2004).

15. Xin, Y. et al. Sulforaphane prevents angiotensin Il-induced cardiomyopathy by activation of Nrf2 via stimulating the Akt/GSK-3ß/Fyn pathway. Redox Biology 15, 405-417 (2018).

16. Czibik, G., Derumeaux, G., Sawaki, D., Valen, G. \& Motterlini, R. Heme oxygenase-1: an emerging therapeutic target to curb cardiac pathology. Basic Res Cardiol 109, 450 (2014).

17. dl-3-n-Butylphthalide prevents neuronal cell death after focal cerebral ischemia in mice via the JNK pathway. Brain Research 1359, 216-226 (2010).

18. Wang, S. et al. DI-3-n-Butylphthalide (NBP): A Promising Therapeutic Agent for Ischemic Stroke. CNSNDDT 17, 338-347 (2018).

19. Zhao, Y. et al. DL-3-n-butylphthalide induced neuroprotection, regenerative repair, functional recovery and psychological benefits following traumatic brain injury in mice. Neurochemistry International 111, 82-92 (2017).

20. Zhang, L. et al. DL-3-n-Butylphthalide, an Anti-Oxidant Agent, Prevents Neurological Deficits and Cerebral Injury Following Stroke per Functional Analysis, Magnetic Resonance Imaging and Histological Assessment. CNR 9, 167-175 (2012).

21. Ku, H.-C., Lee, S.-Y., Wu, Y.-K. A., Yang, K.-C. \& Su, M.-J. A Model of Cardiac Remodeling Through Constriction of the Abdominal Aorta in Rats. JoVE 54818 (2016) doi:10.3791/54818.

22. Kannel, W. B. \& Belanger, A. J. Epidemiology of heart failure. American Heart Journal 121, 951-957 (1991).

23. McArdle, M. \& Hernandez-Vila, E. A. Management of Chronic Venous Disease. Texas Heart Institute Journal 44, 347-349 (2017).

24. Mamas, M. A. et al. Do patients have worse outcomes in heart failure than in cancer? A primary carebased cohort study with 10-year follow-up in Scotland: Outcomes in heart failure and cancer. Eur J Heart Fail 19, 1095-1104 (2017).

25. Lipskaia, L., Chemaly, E. R., Hadri, L., Lompre, A.-M. \& Hajjar, R. J. Sarcoplasmic reticulum Ca ${ }^{2+}$ ATPase as a therapeutic target for heart failure. Expert Opinion on Biological Therapy 10, 29-41 (2010).

26. Erkens, R. et al. Left ventricular diastolic dysfunction in Nrf2 knock out mice is associated with cardiac hypertrophy, decreased expression of SERCA2a, and preserved endothelial function. Free Radical Biology and Medicine 89, 906-917 (2015).

27. Kaspar, J. W., Niture, S. K. \& Jaiswal, A. K. Nrf2:INrf2 (Keap1) signaling in oxidative stress. Free Radical Biology and Medicine 47, 1304-1309 (2009).

28. Zhou, S., Sun, W., Zhang, Z. \& Zheng, Y. The Role of Nrf2-Mediated Pathway in Cardiac Remodeling and Heart Failure. Oxidative Medicine and Cellular Longevity 2014, 1-16 (2014).

29. Li, S. et al. Nrf2 Deficiency Exaggerates Doxorubicin-Induced Cardiotoxicity and Cardiac Dysfunction. Oxidative Medicine and Cellular Longevity 2014, 1-15 (2014). 
30. Li, H. et al. Adiponectin ameliorates hyperglycemia-induced cardiac hypertrophy and dysfunction by concomitantly activating Nrf2 and Brg1. Free Radical Biology and Medicine 84, 311-321 (2015).

\section{Figures}

A
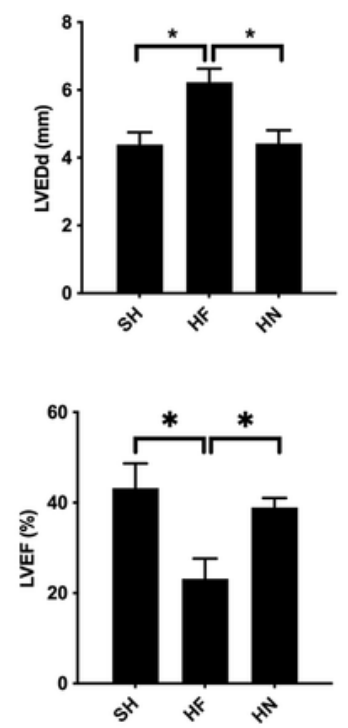

C

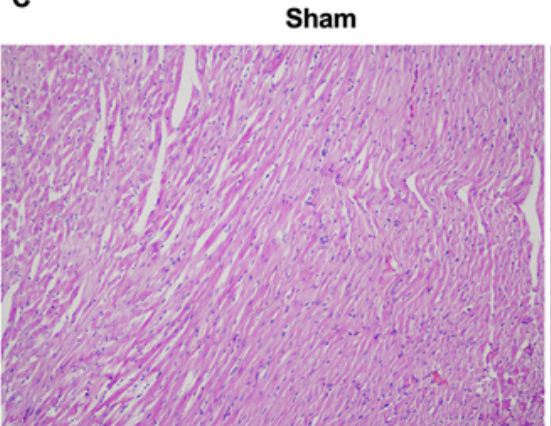

。

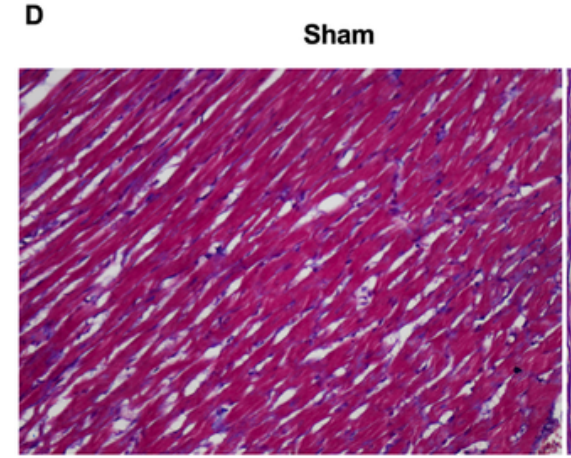

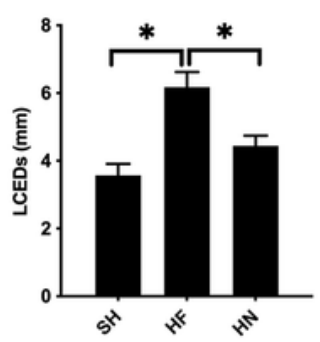

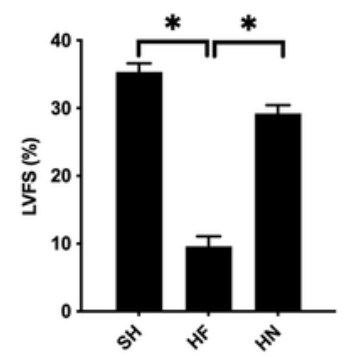

B
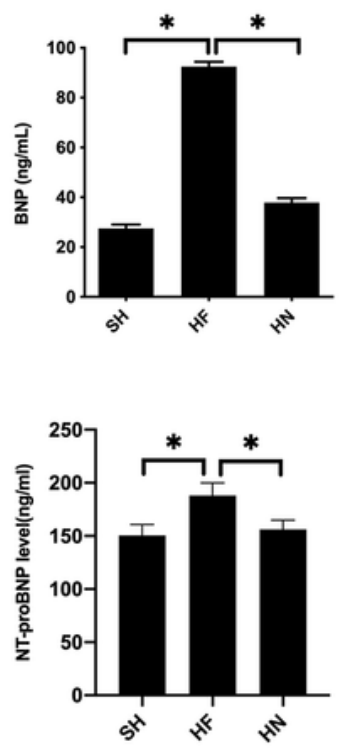

HF

HN
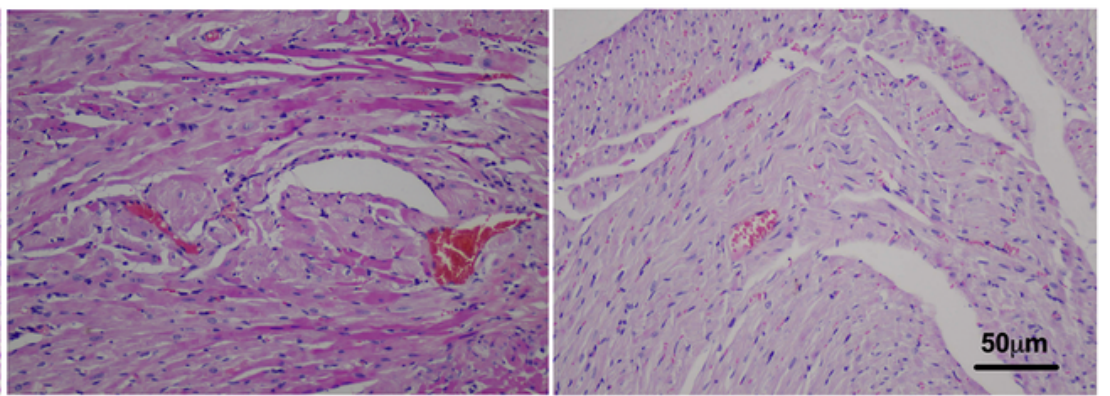

HF

HN
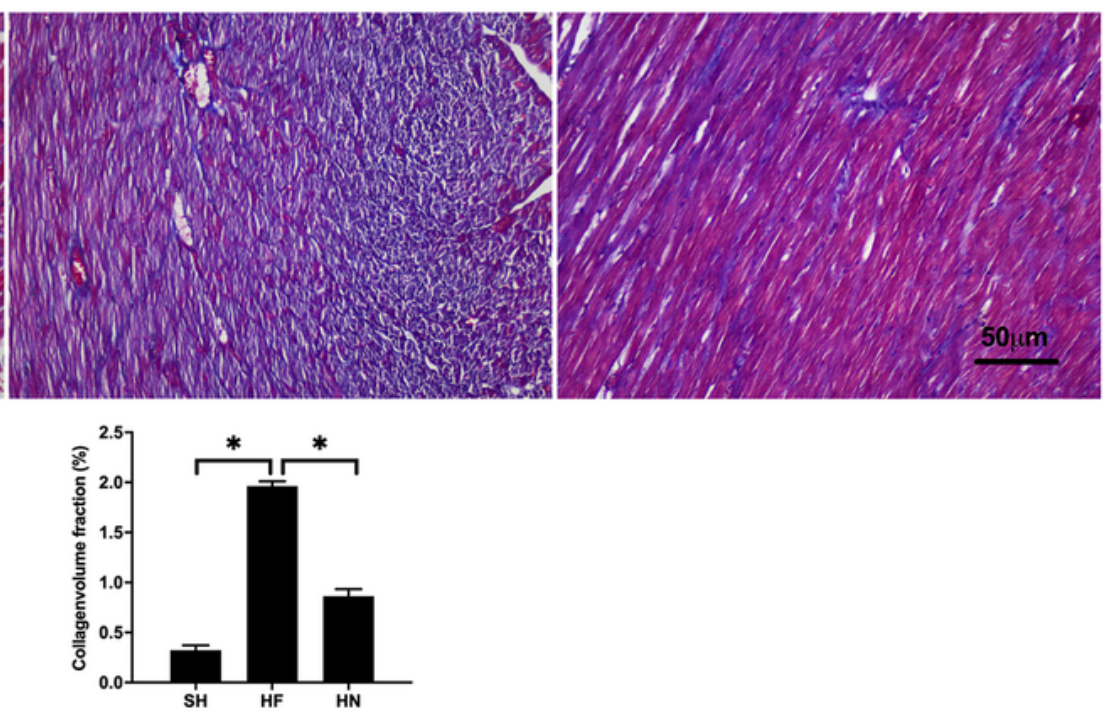

Figure 1 
NPB promoted heart function of Heart failure mice and relieved myocardial injury (A) The Echocardiographic results, (B) The ELISA results of BNP level and NT-proBNP level, (C) Pathological damages examined by HE staining (scale bar $=50 \mu \mathrm{m}$ ), (D) Masson staining (scale bar $=50 \mu \mathrm{m}$ ), ' ${ }^{\prime}, \mathrm{p} p<$ 0.05 .
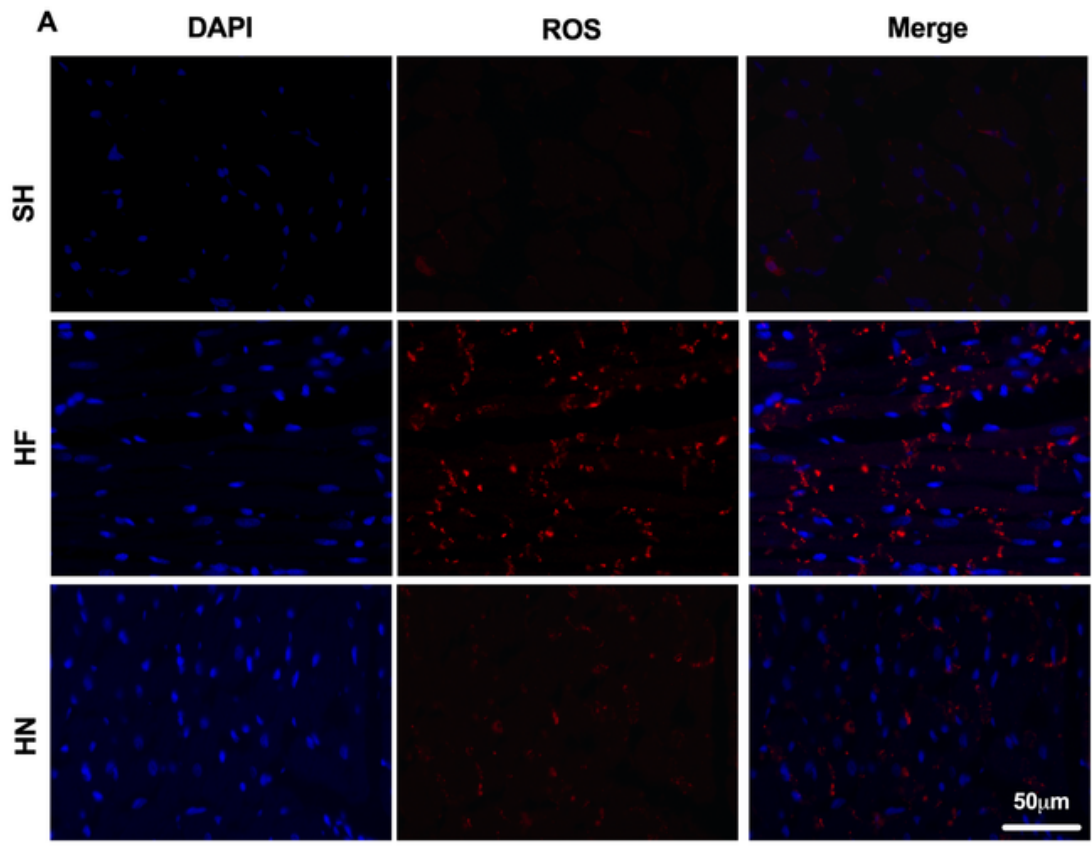

C

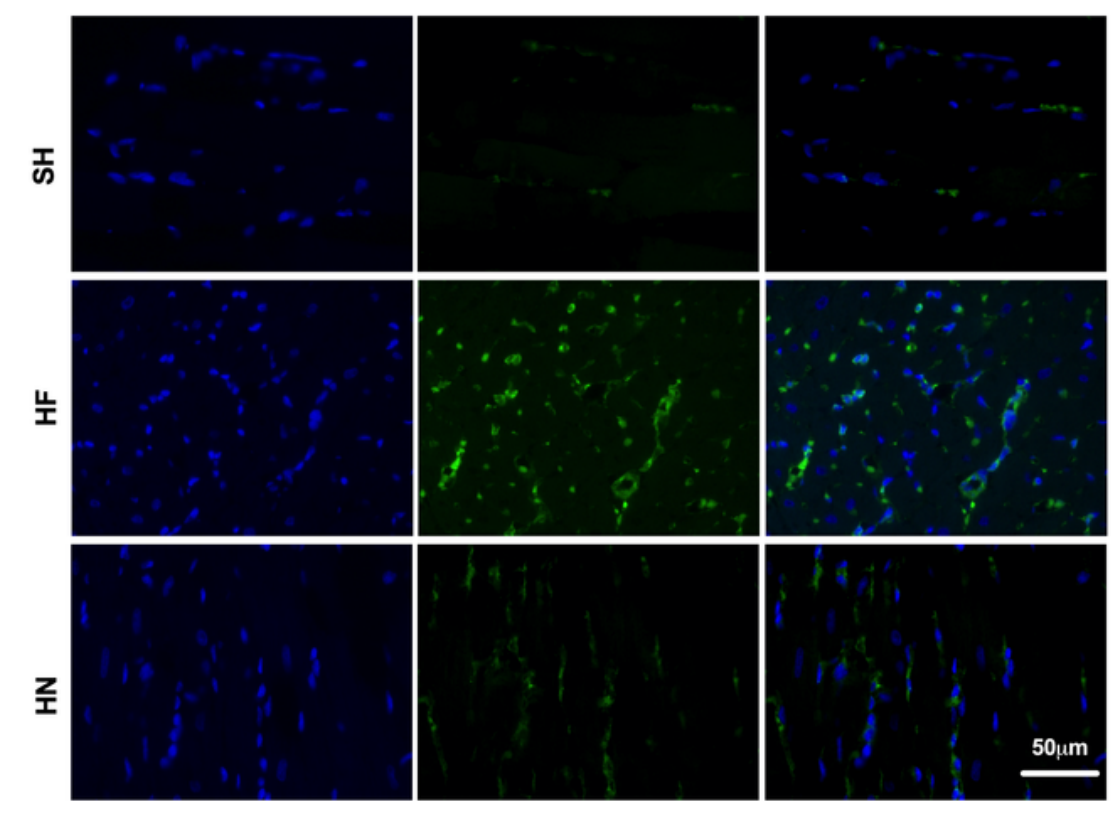

B
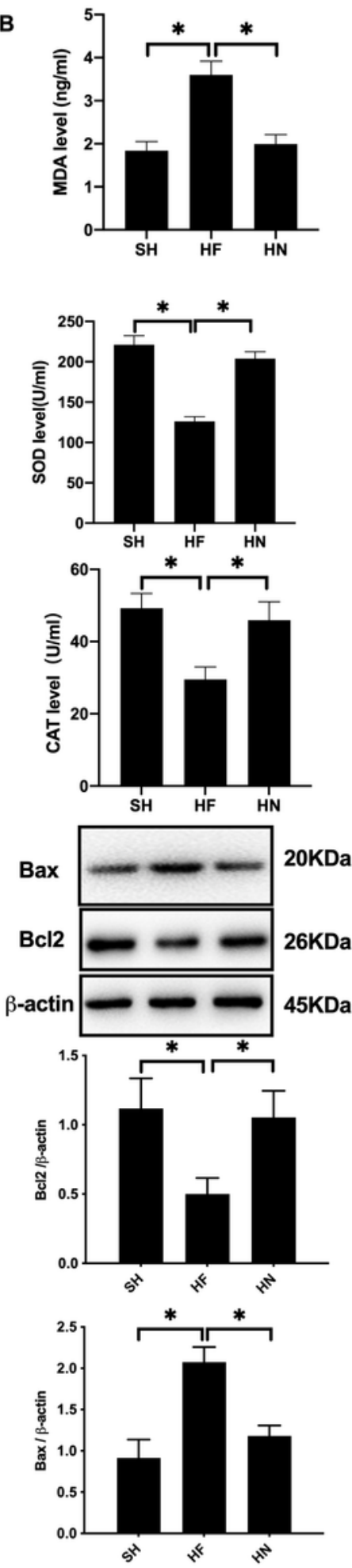

Figure 2 
NPB alleviated oxidative stress injury and decrease the apoptosis of cardiomyocyte of Heart Failure mice (A) The levels of ROS in mice was examined by IFA (scale bar $=20 \mu \mathrm{m})$, (B) The level of MDA, SOD and CAT of plasma examined by ELISA assay, (C) The TUNEL staining results demonstrated the rat myocardial apoptosis (scale bar $=20 \mu \mathrm{m}$ ), (D) Bax and Bcl-2 identified using western blotting, ' ${ }^{\prime}$ ', $\mathrm{p}<0.05$.
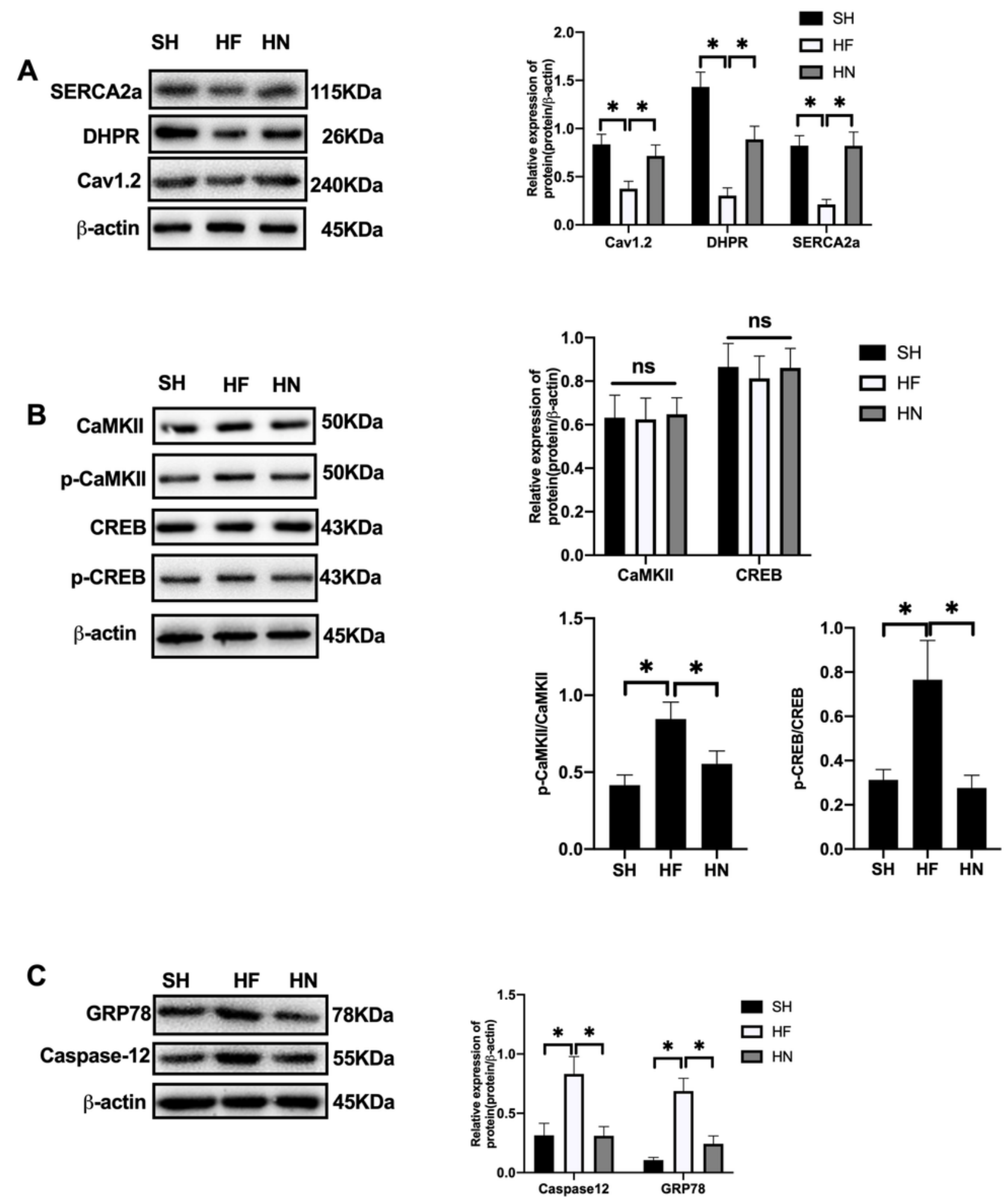

Figure 3 
NPB treatment could enhance Ca2+-SERCA2a and inhibit ERS response in HF mice (A) Western Blotting results showed the expression of SERCA2a, calcium-regulated proteins, (B) Western Blotting results showed the level phosphorylation of CaMKII and CREB, (C) Western Blotting results showed the ERSassociated regulatory proteins. ' ${ }^{\prime}, \mathrm{p} p<0.05$.

A

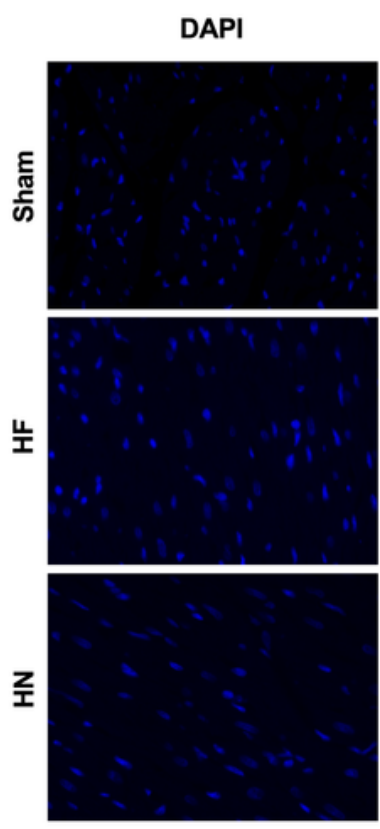

B

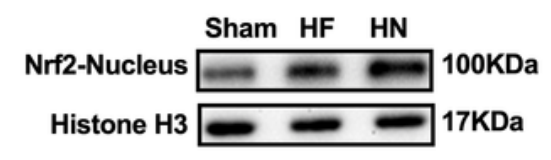

C

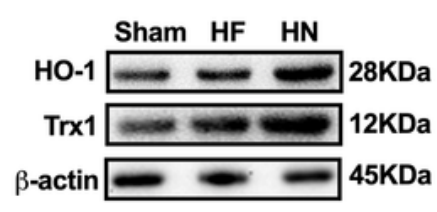

Nrf2
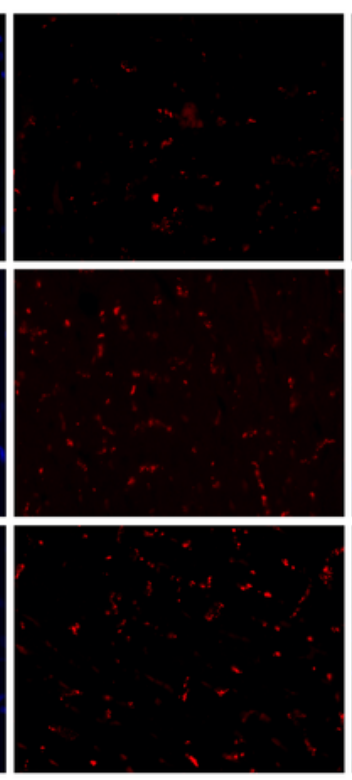

Merge
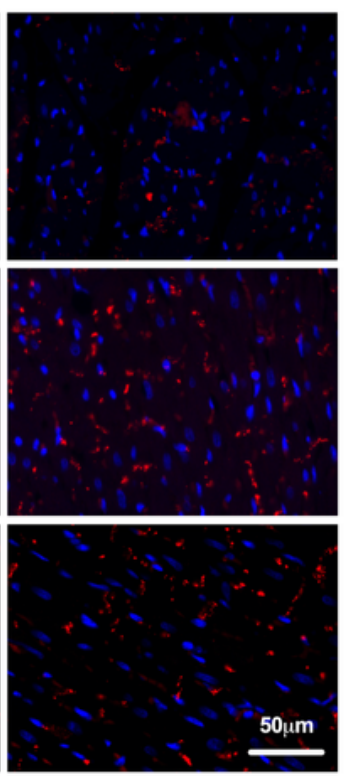

Figure 4

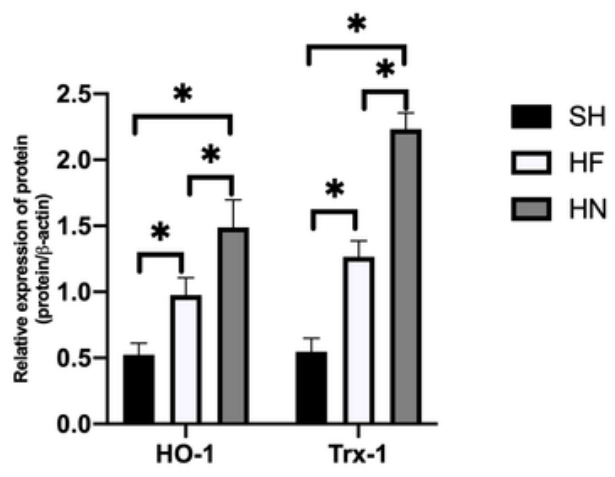


NPB treatment stimulates Nrf2/HO-1 pathway in Heart falure mice (A) The results of IFA showed the Nrf2 expression in mice' myocardial nucleus and cytoplasm detected by IFA (scale bar $=20 \mu \mathrm{m}$ ), (B) The result of western blot demonstrated the expression of Nrf2, HO-1 and Trx1) ' $*$ ', $\mathrm{p}<0.05$.

A

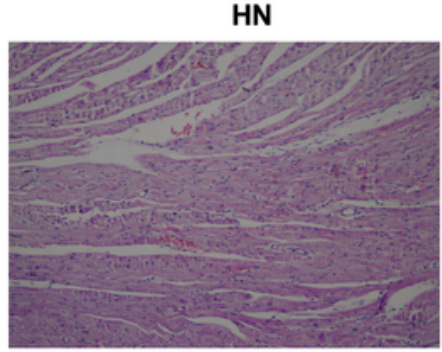

HNK

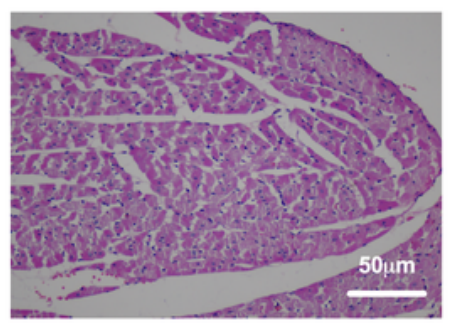

B

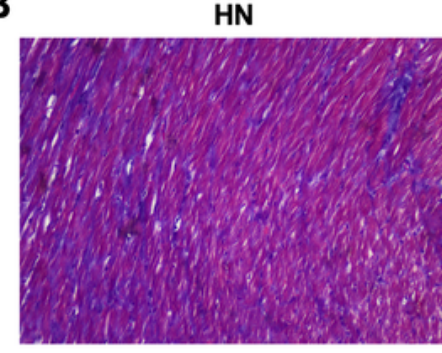

C

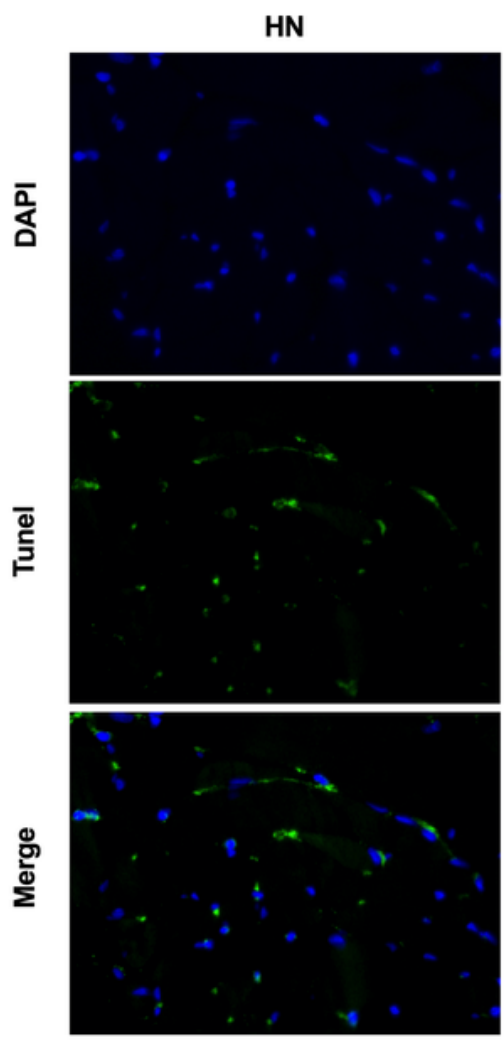

HNK

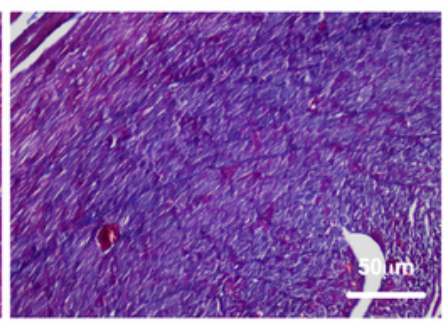

D
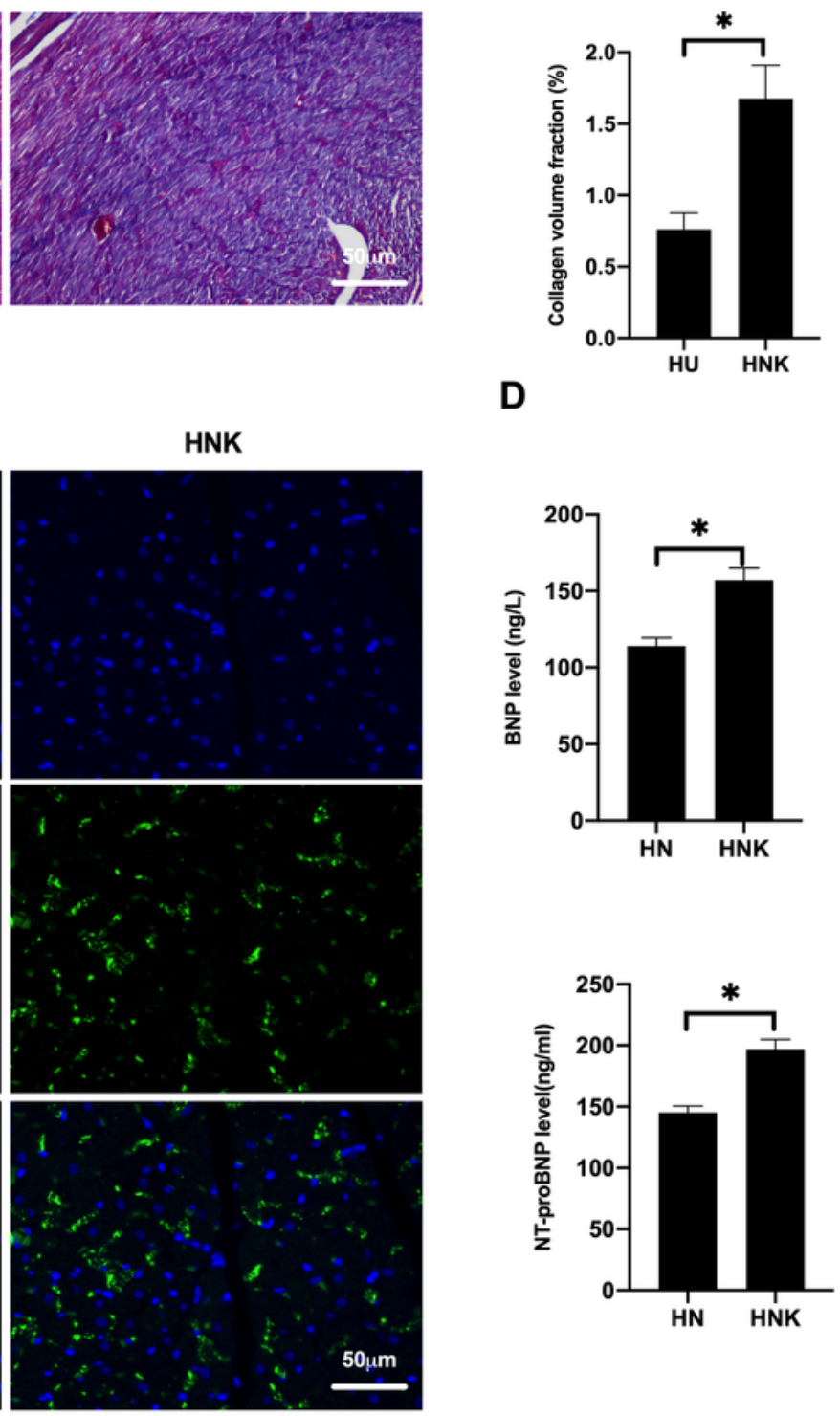

Figure 5

The therapeutic effect of NPB treatment on myocardium in HF mice was partially reversed by CaMKII antagonist (A) The results of HE staining showed the pathological changes in myocardial tissues (scale 
bar $=50 \mu \mathrm{m}),(B)$ The results of Masson staining showed the tissue fibrosis (scale bar $=50 \mu \mathrm{m}),(\mathrm{C})$ The results of TUNEL staining show the myocardial apoptosis rate (scale bar $=20 \mu \mathrm{m}$ ), (D) The natriuretic peptide levels were examined by ELISA, ' ${ }^{\prime}, \mathrm{p}<0.05$.

A
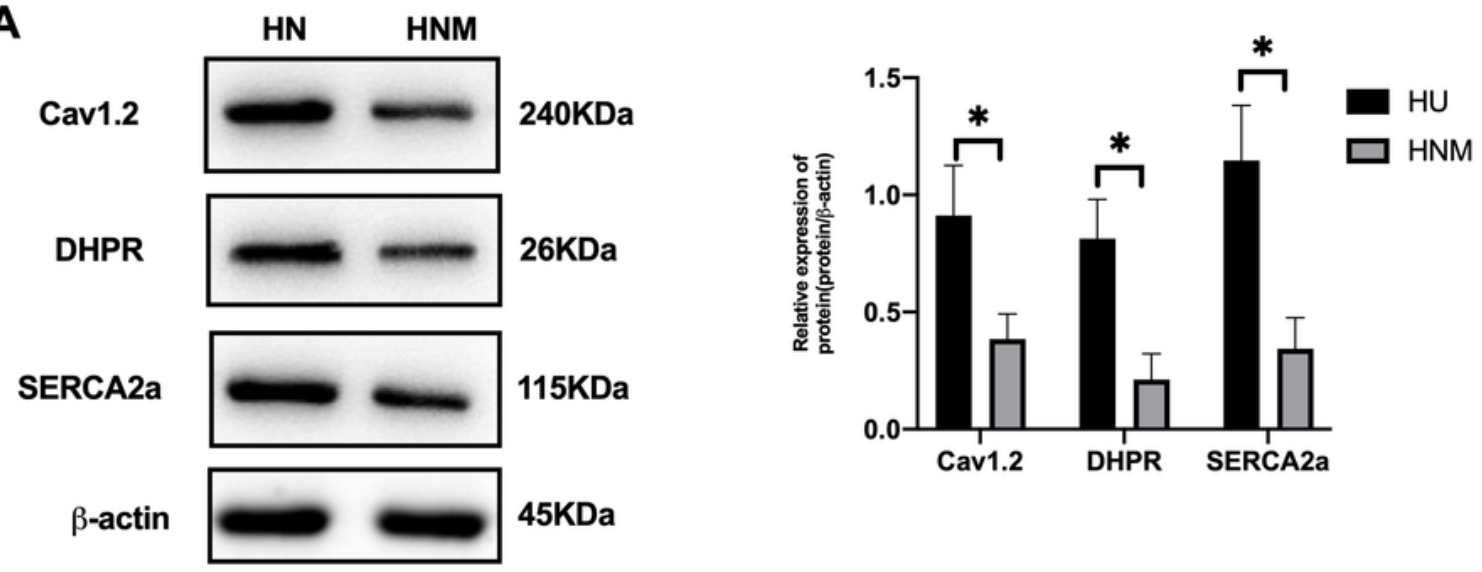

B
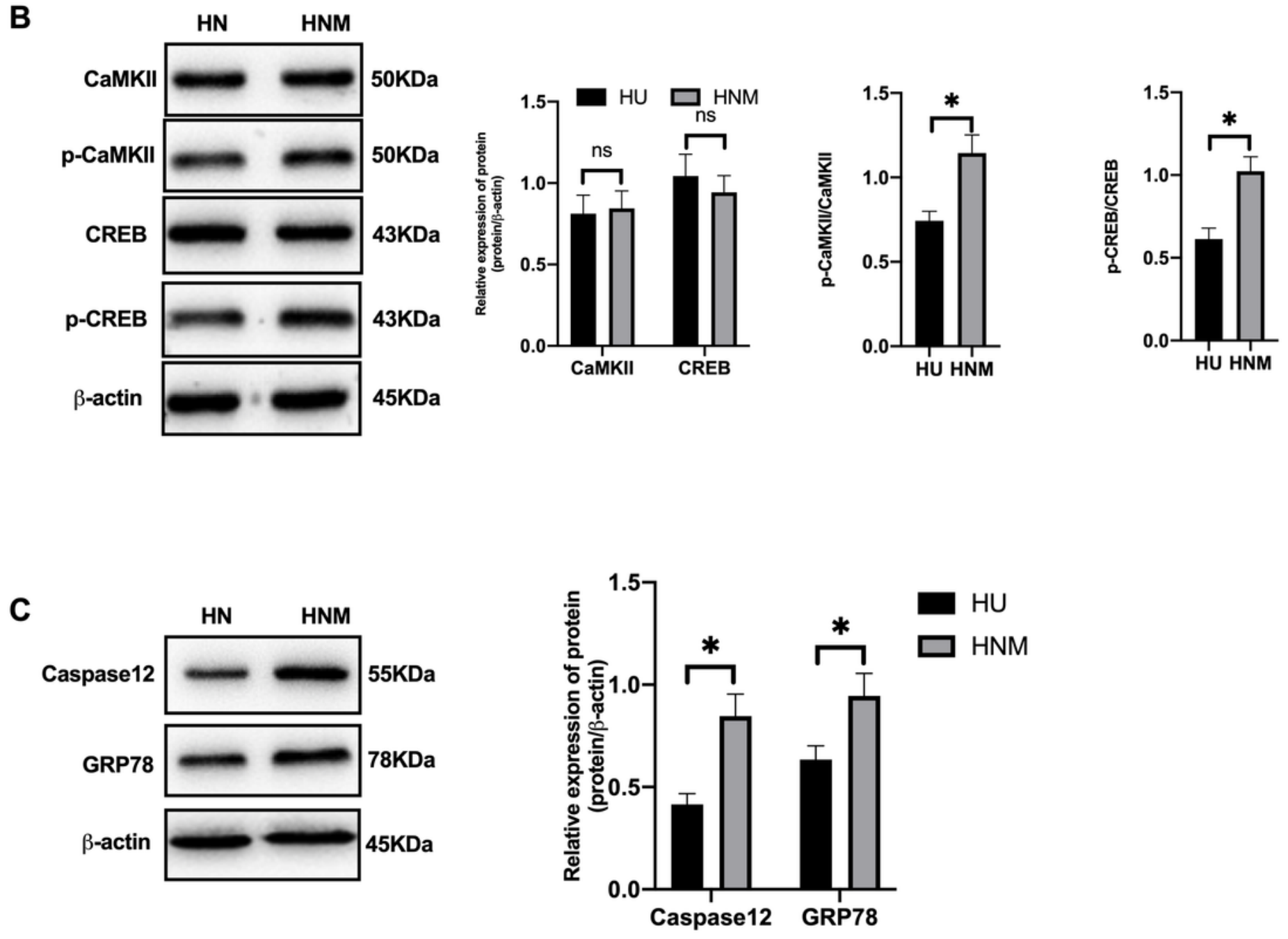

Figure 6

Nrf2 inhibitor blocks the activation of Ca2+-SERCA2a under NBP treatment (A) Western blotting results showed the expression of SERCA2a, calcium-regulated proteins, (B) Western blotting demonstrated the 
phosphorylation level of CaMKII and CREB, (C) Western Blotting results showed the ERS-associated regulatory proteins. ${ }^{*}$ ', $\mathrm{p}<0.05$. 